\title{
Demonstration of Automatic Impedance-Matching and Constant Power Feeding to and Electric Helicopter via Magnetic Resonance Coupling
}

\author{
Masato Yamakawa1, Kohei Shimamura², Kimiya Komurasaki³, Hiroyuki Koizumi4 \\ ${ }^{1}$ Department of Aeronautics and Astronautics, The University of Tokyo, Tokyo, Japan \\ ${ }^{2}$ Department of Engineering Mechanics and Energy, University of Tsukuba, Ibaraki, Japan \\ ${ }^{3}$ Department of Advanced Energy, The University of Tokyo, Chiba, Japan \\ ${ }^{4}$ Research Center for Advanced Science and Technology, The University of Tokyo, Tokyo, Japan \\ Email: shimamura@kz.tsukuba.ac.jp
}

Received 7 April 2014; revised 18 May 2014; accepted 2 June 2014

Copyright (C) 2014 by authors and Scientific Research Publishing Inc.

This work is licensed under the Creative Commons Attribution International License (CC BY). http://creativecommons.org/licenses/by/4.0/

c) (i) Open Access

\begin{abstract}
Wireless power transfer (WPT) from a transmitter resonator on the ground to an electrically powered miniature heli-copter was attempted to demonstrate WPT using magnetic resonance coupling to an object moving in 3D space. The transmission efficiency was optimized by automatic impedance matching for different flight attitudes: a maximum flight altitude of $590 \mathbf{~ m m}$ was achieved. Furthermore, an estimation method of transmission efficiency using only the properties on the transmitter side was proposed, with transmission power regulated as constant against the change in the coupling coefficient.
\end{abstract}

Keywords

Wireless Power Transfer, Power Regulation, Resonator, Flying Object

\section{Introduction}

Since wireless power transfer (WPT) was proposed by Nikola Tesla, WPT applications have increasingly attracted interest. Types of WPT are categorized as radiative and non-radiative forms, such as microwaves, lasers, electromagnetic induction, and magnetic resonance coupling (MRC). Earlier research shows that WPT via MRC has the considerably higher transmission efficiency than microwave transmission at the same distance as the 
transmission coil diameter. In 2007, an MIT group demonstrated WiTricity, the first MRC system, which conducted $1 \mathrm{~m}$ distance transmission with 90\% efficiency [1] [2]. That year, the Nevada Lightning Laboratory achieved $800 \mathrm{~W}$ power transfers over $5 \mathrm{~m}$ distance [3]. In 2009, Sony group achieved long-distance transmission with a relay coil between the resonators [4]. The benefits of MRC include the considerable practical applications of WPT to electric vehicles. Recently, interest has also arisen in MRC applications for mobile devices. In 2009, Qualcomm demonstrated a wireless charging technology for eZONE mobile devices [5]. A WPT demonstration for stationary devices was conducted. However, many difficulties persist in transmission for moving objects.

To develop WPT technology that is applicable to mobile devices, it is first necessary to manufacture a small, light, and highly efficient receiver for use with the transmission system. Furthermore, automatic impedancematching and load-power control systems are needed because the relative positions of the transmitter and receiver coils can change. For an impedance matching technique, some researchers have used a circuit with a tuning capacitor and inductor, controlling several condensers in the circuit [6].

For our previous study, a small helicopter with a light-receiving resonator (3.8 g) was developed using a resonance frequency $\omega$ of $40.68 \mathrm{MHz}$ [7]. A four-coil system comprising an excitation coil, transmitter resonator coil, receiver resonator coil, and pickup coil was adopted. Its transmission efficiency of $50 \%$ was achieved by one-side impedance matching and $100 \mathrm{~mm}$ altitude flight. Impedance was controlled on the transmitter side by adjusting the distance between the excitation coil and the transmitter resonator, avoiding the changes in its resonant frequency. However, to minimize the receiver weight on the helicopter, impedance on the receiver side was not matched.

For this study, a flight attitude of $590 \mathrm{~mm}$ was achieved by optimizing the transmitter designing. In addition, a method for estimating the transmission efficiency was proposed to maintain the transmission power as constant against the change in the coupling coefficient.

\section{Sizing of Resonator and Coils}

To achieve a higher flight altitude than that reported from an earlier study, the transmission system was redesigned. An open-source software package of moment method, 4NEC2, was used to estimate the coupling coefficient between the coils [8]. According to this software, the relation between the total length of coil $l_{\mathrm{c}}$ and wavelength $\lambda$ is expected to satisfy $\lambda / l_{\mathrm{c}}<0.15$. However, it is difficult to satisfy this relation at $\omega=40.68 \mathrm{MHz}$. Therefore, $\omega$ was changed to $13.56 \mathrm{MHz}$. The transmitter resonator was enlarged to $600 \mathrm{~mm}$ diameter while the receiver size remained the same. The method of one-side impedance matching on the transmitter side was the same as that used in our previous study [7]. A vector network analyzer (MS2035B; Anritsu Corp.) with a calibration material (3750R; Anritsu Corp.) was used to measure the reflected power. Table 1 presents resonator specifications. The skin depth at this frequency is about $0.02 \mathrm{~mm}$ in copper. Thereby, a copper tube with a 5-mm cross-sectional radius was used for a transmitter resonator. A copper-foil tube was use for a receiver resonator to minimize weight. The receiver resonator was a square of $110 \mathrm{~mm} \times 110 \mathrm{~mm}$ weighing $3.11 \mathrm{~g}$. The equivalent diameter $D_{\mathrm{R}}$ of a circular resonator is the same as that of $124 \mathrm{~mm}$ rectangular resonator. Because the voltage in the excitation coil and the resonator increases rapidly with the transmission distance, open-type coils were used as the excitation coil and the transmitter resonators. $D_{\mathrm{T}}$ is the transmitter resonator diameter, which is an open spiral coil like that shown in Figure 1. Transmission power $P_{\mathrm{T}}$ was set to about $5 \mathrm{~W}$, which is $25 \%$ of the nor-

\begin{tabular}{ccc|}
\hline Table 1. Specifications of the resonators. & \\
\hline TYPE & Transmission & Receive \\
\hline Resonance frequency, $\mathrm{MHz}$ & 13.561 & 13.561 \\
Size, $\mathrm{mm}$ & $D_{\mathrm{T}}=600$ & $110 \times 110 D_{\mathrm{R}}=62.124$ \\
Number of turns & 2.25 & 1 \\
Quality factor & 197 & 313 \\
Capacitance of a capacitor, pF & Open & 430 \\
Weight, g & - & 3.11 \\
Diameter of coils, mm & 10 & 3 \\
\hline
\end{tabular}



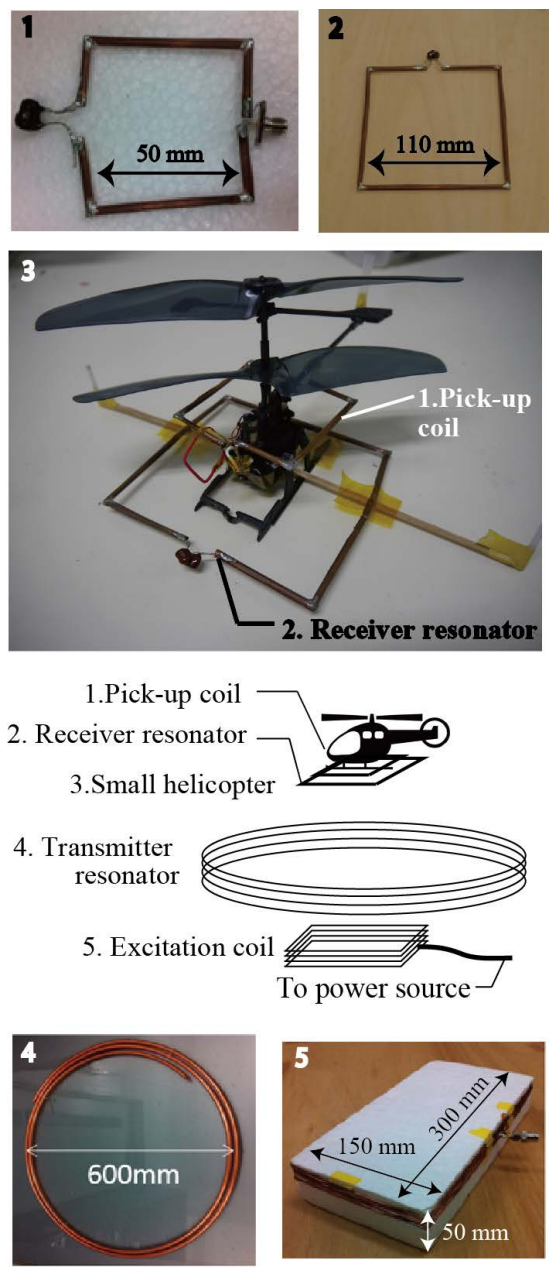

Figure 1. Wireless power transfer system for the small helicopter.

mal rated power of the helicopter's motor. The nominal transmission distance $l$ was set at $550 \mathrm{~mm}$, which is twice as large as the average resonator diameter $\sqrt{D_{\mathrm{T}} D_{\mathrm{R}}}$ of $1930 \mathrm{~mm}$. Here the non-dimensional distance $l^{\prime}$ is introduced as

$$
l^{\prime}=\frac{l}{\sqrt{D_{\mathrm{T}} D_{\mathrm{R}}}}
$$

The optimum impedance ratio $r_{\mathrm{T}}$ is a function of $Q_{\mathrm{T}}, Q_{\mathrm{R}}, r_{\mathrm{R}}$, and the coupling-coefficient $k$ as

$$
r_{\mathrm{T}}=\frac{k^{2} Q_{\mathrm{T}} Q_{\mathrm{R}}}{1+r_{\mathrm{R}}}+1
$$

where $Q$ is a quality factor defined as $Q=\omega L / R$. Subscripts $\mathrm{T}$ and $\mathrm{R}$ respectively denote the transmitter side and receiver side. Impedance ratios $r_{\mathrm{T}}$ and $r_{\mathrm{R}}$ are defined respectively as $r_{\mathrm{T}}=Z_{\mathrm{src}} / R_{\mathrm{T}}$ and $r_{\mathrm{T}}=Z_{\mathrm{load}} / R_{\mathrm{R}}$. Subscripts src and the load respectively denote the power source and external load. At $l=0, k$ approaches unity and $r_{\mathrm{T}, \mathrm{opt}}$ increases to 37. In preliminary testing, the impedance ratio of transmission side $r_{\mathrm{T}}$ was measured to confirm that the excitation coil can satisfy Equation (2). $r_{\mathrm{T}}$ is tunable by adjusting the coupling coefficient between the excitation coil and the transmitter resonator $k_{\mathrm{ET}}$, as [9]

$$
r_{\mathrm{T}}=\frac{Q_{\mathrm{T}} Q_{\mathrm{E}} k_{\mathrm{ET}}^{2} R_{\mathrm{E}}}{R_{\mathrm{E}}+Z_{\mathrm{src}}}
$$


Subscript E denotes the excitation coil. To design the excitation coil, the voltage and the current between the excitation coil and the transmitter resonator were analyzed using LTspice [10]. Table 2 presents excitation coil specifications. A pick-up coil was made of a copper-foil tube that was the same as the receiver resonator. The helicopter load resistance was assumed as $5 \Omega$ at $P_{\mathrm{T}}=5 \mathrm{~W}$. In this study, the receiver impedance was matched at $l=500 \mathrm{~mm}$ and fixed. Here, $k_{\mathrm{ET}}$ was measured in the same manner as $k$. Figure 2 portrays the measured impedance ratio on the transmitter side as a function of distance between the centers of respective coil. Results show that $r_{\mathrm{T}}$ satisfied the relation of Equation (2) within the movable range of the coil. Finally, Figure 3 presents the

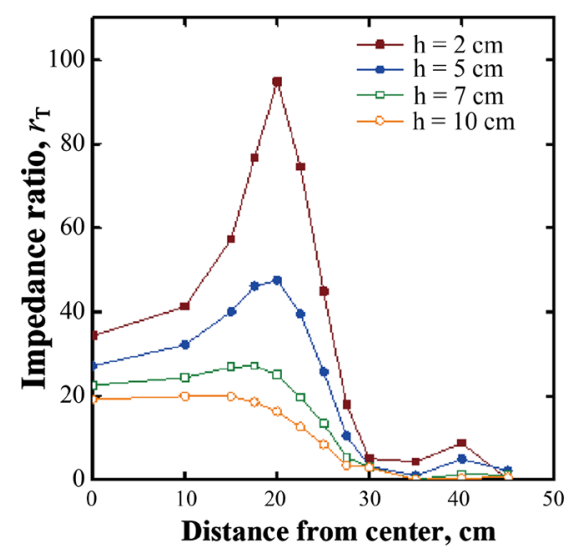

Figure 2. Measured $r_{\mathrm{T}}$ on the transmitter side as a function of distance between the centers of each coil.

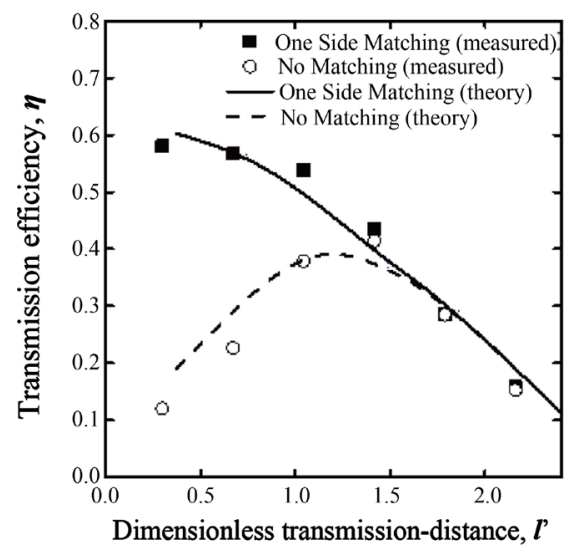

Figure 3. Measured and computed $\eta$ with and without impedance matching as a function of dimensionless transmission-distance $l$ '.

Table 2. Specifications of the pick-up and excitation coil.

\begin{tabular}{ccc}
\hline Type of coil & Pick-up & Excitation \\
\hline Resonance frequency, $\mathrm{MHz}$ & 13.561 & 13.561 \\
Size on a side, $\mathrm{mm}$ & $50 \times 50$ & $150 \times 300$ \\
Diameter of wire, $\mathrm{mm}$ & 3.0 & 1.0 \\
Capacitance of a capacitor, $\mathrm{pF}$ & 1100 & Open \\
Material & Copper & Copper \\
Number of turns & 1 & 5 \\
Unloaded $Q$ & - & 500 \\
Resistance, $\Omega$ & - & 1.25 \\
\hline
\end{tabular}


measured and computed power transmission efficiency $\eta$ with and without impedance matching as a function of $l^{\prime}$. Measured values showed good agreement with the computed values. In addition, the present transmission system achieved high transmission efficiency in the low-altitude region up to $45 \%$ by appropriate impedance matching.

\section{Flight Demonstration of a Toy Helicopter}

\subsection{Impedance Matching by Power-Reflection Monitoring}

Without impedance matching, $\eta$ has a peak around the designed nominal altitude. It is difficult for the helicopter to hover at a certain altitude where $\eta$ increased with $l$ '. In this sense, impedance matching is unavoidable for the helicopter to conduct a safe liftoff and landing. $r_{\mathrm{T}, \mathrm{opt}}$ was obtained by minimizing the reflection power $P_{\mathrm{R}}$. This control method is extremely simple.

\subsection{Experimental Setup}

A small electric helicopter with the receiver system was used in the same manner as in our previous study. As Figure 1 shows, the receiver system consists of a receiver resonator, a pick-up coil and a diode-bridge rectifier circuit. Figure 4 and Figure 5 respectively show the automatic impedance-matching system and the control flow diagram. Input power and reflected power are monitored and sent as a reference signal to a microcomputer (H8-3694F; Renesas Electronics Corp.), which controls the actuator. At several connection points where DC current flows, RC low-pass filters were used to remove the high-frequency noise. The pulse-width modulation

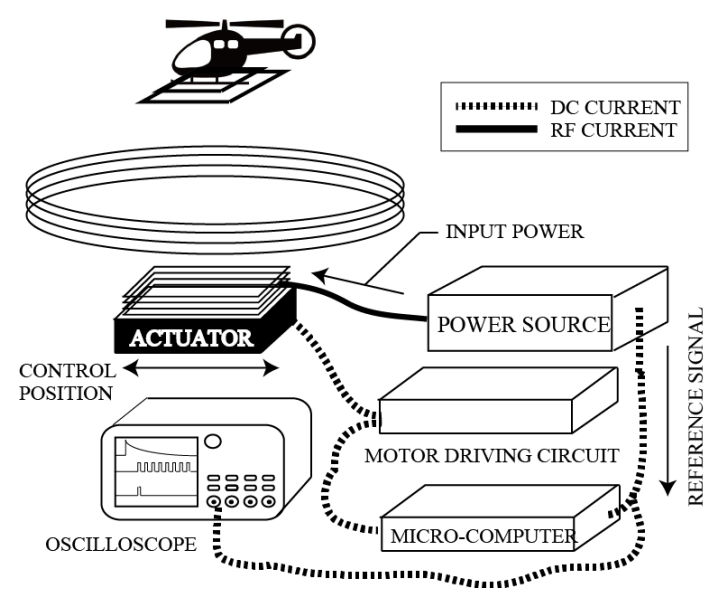

Figure 4. Automatic impedance-matching system for a flight demonstration.

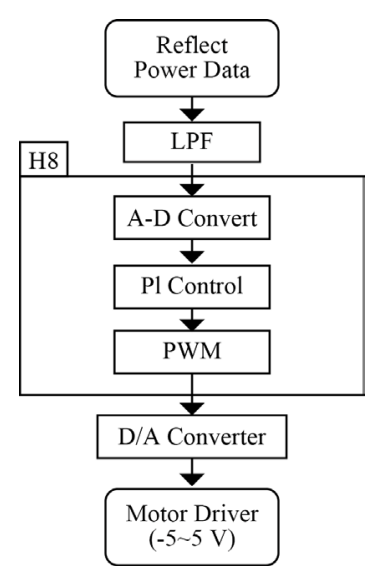

Figure 5. Flow of the automatic-control system. 
(PWM) signal is sent from the micro-computer to the actuator via a motor-driver circuit. An RF power source (T161-5613 HA; Thamway, Corp.) with maximum power of $400 \mathrm{~W}$ and RF frequency of $13.56 \mathrm{MHz}$ was used. It enables us to monitor the input and the reflection as well as external control of the output power.

\subsection{Results}

As Figure 6 shows, the helicopter reached $590 \mathrm{~mm}$ and showed flight with impedance matching. This altitude was higher than the $500 \mathrm{~mm}$ designed distance. Without impedance matching hovering was difficult. Figure 7 shows the history of reflected power before and after impedance matching. At $t=0 \mathrm{~s}, P_{\mathrm{T}}=8 \mathrm{~W}$ and $P_{\mathrm{R}}=3 \mathrm{~W}$. The excitation coil position was out of control. When impedance matching started at $10 \mathrm{~s}, P_{\mathrm{R}}$ was $0.2 \mathrm{~W}$.

\section{Constant Power Feeding Demonstration}

\subsection{Method of $\eta$ Estimation}

When $r_{\mathrm{R}}$ is given, $\eta$ can be estimated using parameters on the transmitter side. Considering the energy losses in excitation and pickup coils, $k$ is expressed as

$$
\eta=\frac{4 k^{2} Q_{\mathrm{T}} Q_{\mathrm{R}} r_{\mathrm{T}} r_{\mathrm{R}}}{\left[k^{2} Q_{\mathrm{T}} Q_{\mathrm{R}}+\left(1+r_{\mathrm{T}}\right)\left(1+r_{\mathrm{R}}\right)\right]^{2}} \eta_{\text {src }} \eta_{\text {load }}
$$

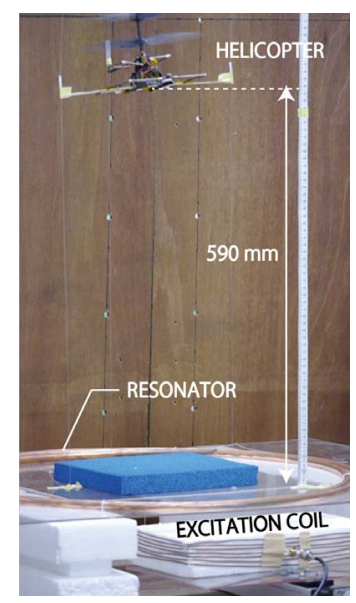

Figure 6. Automatic impedance-matching system for a flight demonstration.

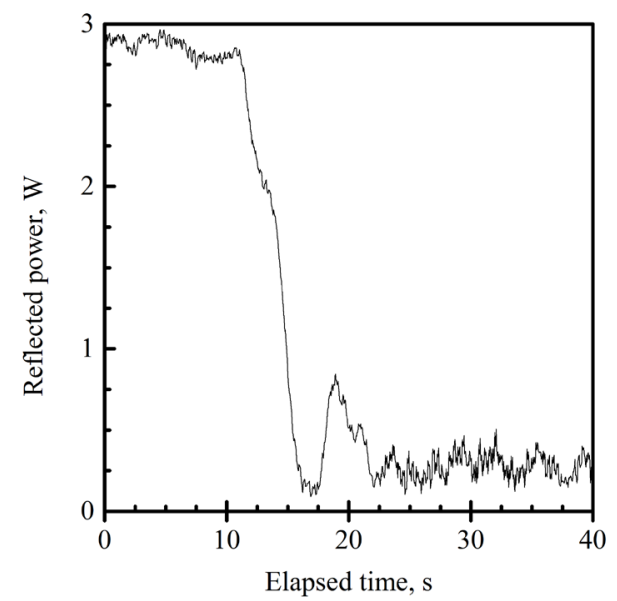

Figure 7. History of reflection power. At $t=10 \mathrm{~s}$ impedance matching started. 
where $\eta_{\text {src }}=Z_{\text {src }} /\left(R_{\mathrm{E}}+Z_{\text {src }}\right)$ and where $\eta_{\text {load }}=Z_{\text {load }} /\left(R_{\mathrm{P}}+Z_{\text {load }}\right)$. The subscript load denotes the load of the light bulb. $k$ is expressed with $S_{11}=\sqrt{P_{R} / P_{T}}$ as

$$
k=\sqrt{\frac{1+r_{\mathrm{R}}}{Q_{\mathrm{T}} Q_{\mathrm{R}}} \frac{\left(1-S_{11}\right)\left(1+r_{\mathrm{T}}\right)-2 \eta_{\mathrm{src}}}{1-S_{11}-2 \eta_{\mathrm{src}}}} .
$$

Substituting Equation (5) into Equation (4), $\eta$ yields

$$
\eta=\frac{\left(2 \eta_{\text {src }}-\left(1-S_{11}\right)\right)\left(2 \eta_{\text {src }}-\left(1+r_{\mathrm{T}}\right)\left(1-S_{11}\right)\right) r_{\mathrm{R}}}{\left(1+r_{\mathrm{R}}\right) r_{\mathrm{T}} \eta_{\mathrm{src}}} \eta_{\text {load }}
$$

Actually, $\eta_{\text {load }}$ is usually approximately unity. Also $\eta_{\mathrm{src}}$ and $r_{\mathrm{T}}$ are given. Consequently, $\eta$ can be estimated by monitoring and $r_{\mathrm{T}}$ and $S_{11}$.

\subsection{Experimental Setup}

Figure 8 portrays the constant power supply demonstration system. The reference signals of the input and reflected power sent to the microcomputer were used to estimate the transmission efficiency. The microcomputer was the same as that used in the flight demonstration. However, it was used not for impedance matching but for input power regulation from the power source to maintain $10 \mathrm{~W}$ at the light bulb in this demonstration. Figure 9 shows a schematic of the pick-up coil and the rectifier circuit. Table 3 shows the pick-up coil and the resonator specifications. A light bulb with rated power consumption of $10 \mathrm{~W}$ was used as the load on the receiver system. The constant power was demonstrated where $R_{\mathrm{T}}$ and $R_{\mathrm{R}}$ were pre-optimized at $l=300 \mathrm{~mm}$. The measured impedance ratio $r_{\mathrm{R}}$ was 9.0 .

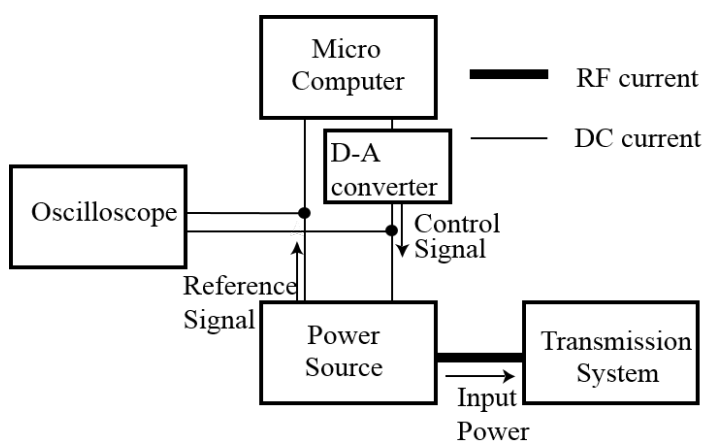

Figure 8. Constant power-feeding demonstration system.

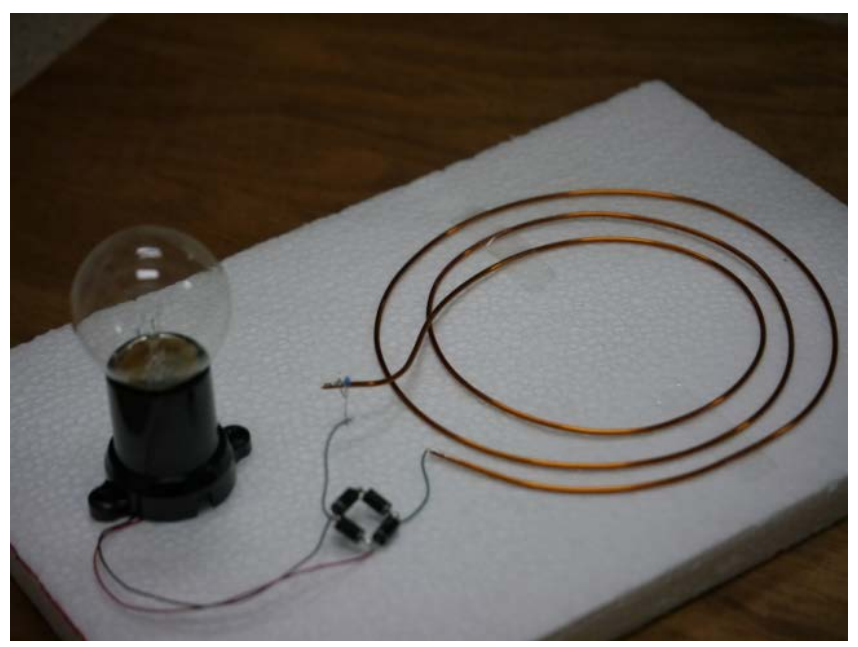

Figure 9. Pick-up coil with the rectifier circuit and the light bulb. 


\subsection{Results}

As Figure 10 shows, with the power control, the light bulb became bright from $l=0 \mathrm{~mm}$ to $300 \mathrm{~mm}$. It was not bright at $l=0 \mathrm{~mm}$ without the power control. Figure 11 portrays the theoretical and estimated transmission efficiency $\eta$ and $S_{11}$ during the demonstration. Estimated $\eta$ agreed well with theoretical $\eta$ within $10 \%$ error. Figure 12 shows the incident power and power consumption during the demonstration. As Figure 12 shows, power consumption was regulated to approximately $10 \mathrm{~W}$. Results show that it is possible to supply constant power with $\eta$ estimation.

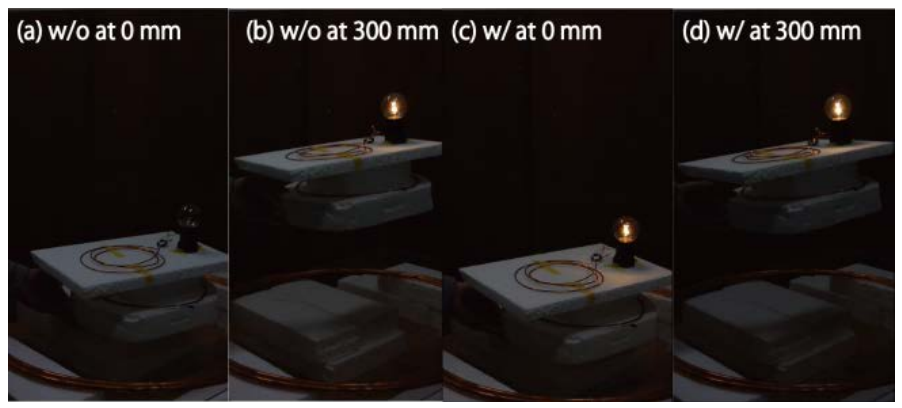

Figure 10. Pick-up coil and light bulb with ((a), (b)) and without ((c), (d)) power control.

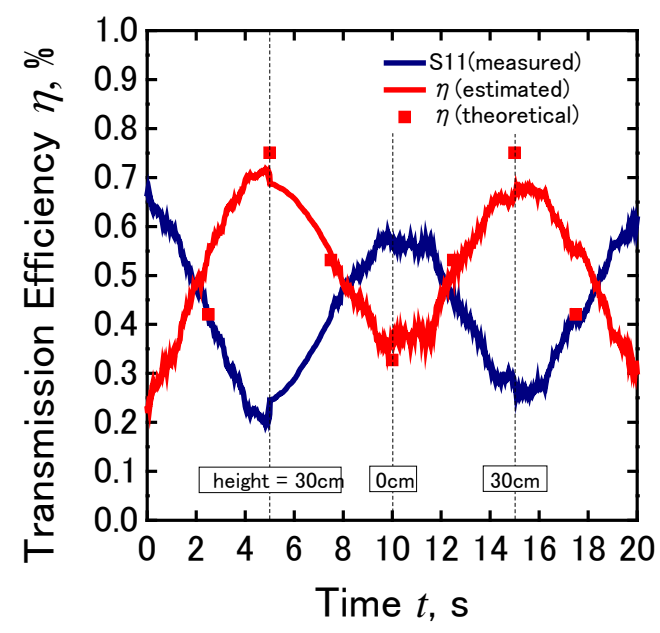

Figure 11. Estimated and theoretical transmission efficiency without power control. $l$ changed from $600 \mathrm{~mm}$ to $0 \mathrm{~mm}$ in $10 \mathrm{~s}$ and from $0 \mathrm{~mm}$ to $600 \mathrm{~mm}$ in next $10 \mathrm{~s}$.

Table 3. Specifications of at the pick-up coil and a resonator.

\begin{tabular}{ccc} 
Type & Pick-up coil & Resonator \\
\hline Resonance frequency, MHz & 13.561 & 13.561 \\
Diameter, mm & 1000 & 1250 \\
Diameter of wire, mm & 0.5 & 1.0 \\
Capacitance, pF & - & 200 \\
Material & Copper & Copper \\
Number of turns & 3 & 1 \\
Q-factor & - & 356 \\
\hline
\end{tabular}




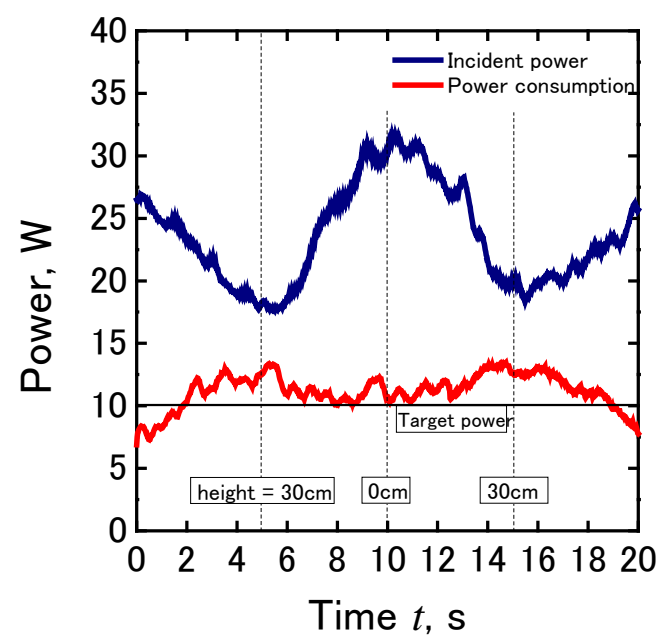

Figure 12. Incident power and power consumption with power control. $l$ changed from $600 \mathrm{~mm}$ to $0 \mathrm{~mm}$ in $10 \mathrm{~s}$ and from $0 \mathrm{~mm}$ to $600 \mathrm{~mm}$ in next $10 \mathrm{~s}$.

\section{Conclusions}

Automatic impedance matching with WPT to a small electric powered helicopter was proposed and demonstrated. Results show that the power transmission efficiency was improved up to $45 \%$ in the high $k$ region. The flight altitude of $590 \mathrm{~mm}$ was achieved with the average resonator diameter $\sqrt{D_{\mathrm{T}} D_{\mathrm{R}}}=1930 \mathrm{~mm}$.

Furthermore, the transmission efficiency estimation method was proposed using only the properties on the transmitter side. By sliding a light bulb from $0 \mathrm{~mm}$ to $600 \mathrm{~mm}$ distant from the transmitter resonator, a constant power supply of the power of $10 \mathrm{~W}$ was demonstrated.

\section{References}

[1] Kurs, A., Joannopoulos, J.D. and Soljacic, M. (2008) Efficient Wireless Non-Radiative Mid-Range Energy Transfer. Annals of Physics, 323, 34-48. http://dx.doi.org/10.1016/j.aop.2007.04.017

[2] Kurs, A., Karalis, A., Moffatt, R., Joannopoulos, J.D., Fisher, P. and Soljacic, M. (2007) Wireless Power Transfer via Strongly Coupled Magnetic Resonance. Science, 317, 83-86. http://dx.doi.org/10.1126/science.1143254

[3] http://www.lightninglab.org/

[4] Hashiguchi, T., Komiyama, S., Mita, H. and Fujimaki, K. (2010) Passive Extender for Wireless Power Transmission Using Magnetic Resonances. IEICE, B-1-25.

[5] http://www.qualcomm.com/media/releases/2010/02/12/qualcomm-demonstrates-evolution-wireless-mobile-computingapplications-and

[6] Beh, T.C., Kato, M., Imura, T., Oh, S. and Hori, Y. (2013) Automated Impedance Matching System for Robust Wireless Power Transfer via Magnetic Resonance Coupling. IEEE Transactions on Industrial Electronics, 60, 3689-3698. http://dx.doi.org/10.1109/TIE.2012.2206337

[7] Koizumi, M., Komurasaki, K., Mizuno, Y. and Arakawa, Y. (2012) Wireless Power Feeding with Strongly Coupled Magnetic Resonance for a Flying Object. Wireless Engineering and Technology, 3, 86-89. http://dx.doi.org/10.4236/wet.2012.32014

[8] Smith, J.L. (2008) Basic NEC with Broadcast Applications. Focal Press, Waltham.

[9] Komaru, T., Koizumi, M., Komurasaki, K., Shibata, T. and Kano, K. (2011) Compact and Tunable Transmitter and Receiver for Magnetic Resonance Power Transmission to Mobile Objects. In: Kim, K.Y., Ed., Wireless Energy Transfer Based on Electromagnetic Resonance: Principles and Engineering Explorations, In Tech, Rijeka, 133-150.

[10] http://www.linear.com/designtools/software/\#LTspice 
Scientific Research Publishing (SCIRP) is one of the largest Open Access journal publishers. It is currently publishing more than 200 open access, online, peer-reviewed journals covering a wide range of academic disciplines. SCIRP serves the worldwide academic communities and contributes to the progress and application of science with its publication.

Other selected journals from SCIRP are listed as below. Submit your manuscript to us via either submit@scirp.org or Online Submission Portal.
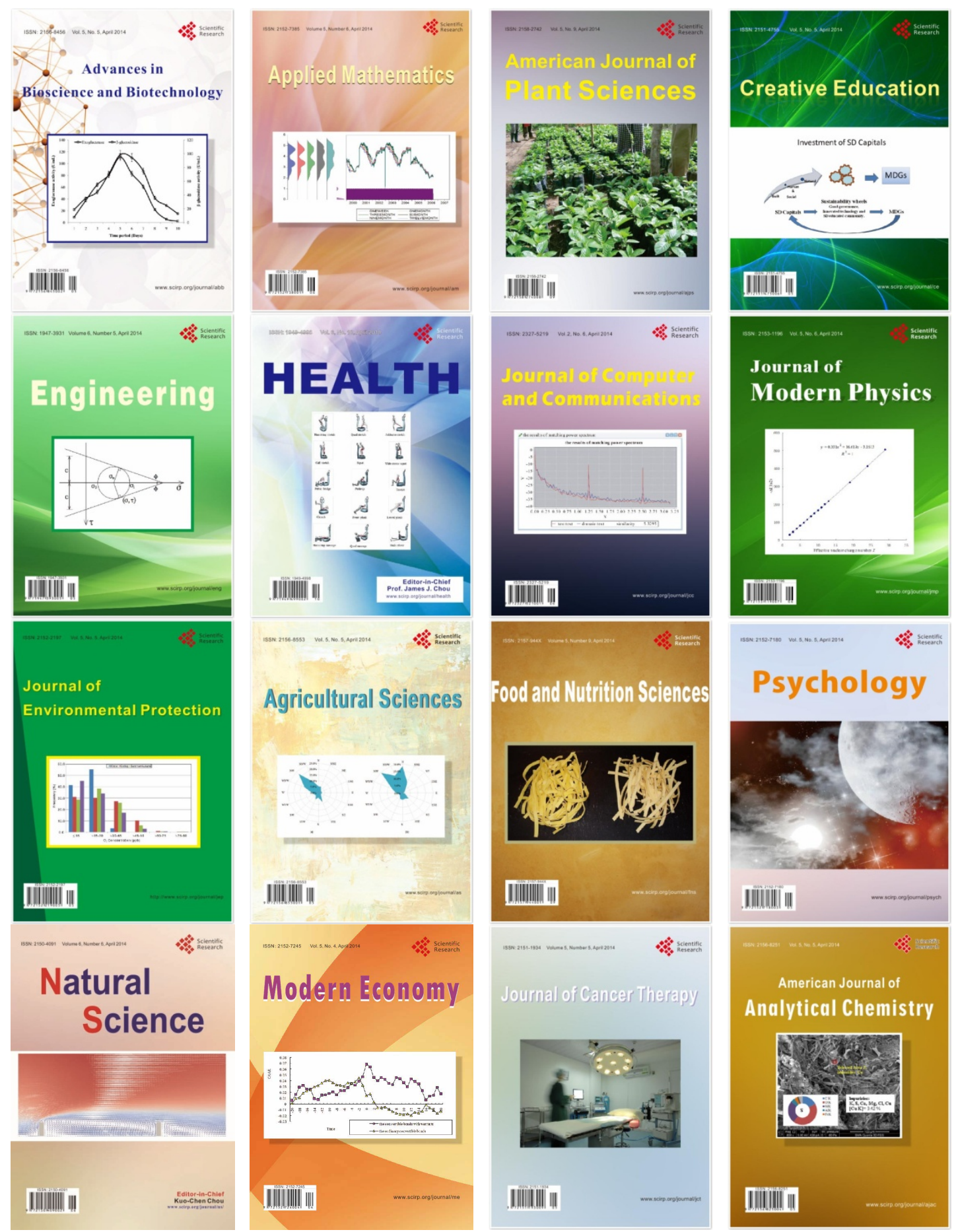